\title{
Ultrasonically Detected Subpleural Lung Consolidations in The Emergency Room: A Case Report
}

\author{
Karen Itzel González Mártinez, ${ }^{1 *}$ Felipe de Jesús Montelongo, ${ }^{2}$ Roberto Emmanuel Islas Ávila, ${ }^{3}$ Giovanni Cerón \\ Solano $^{4}$ \\ ${ }^{1}$ Department of Intensive Care, General Hospital of Ecatepec Las Americas, Mexico \\ ${ }^{2}$ Head of the Intensive Care Unit, General Hospital of Ecatepec Las Américas \\ ${ }^{3}$ Department of Intensive Care, General Hospital of Ecatepec Las Americas, Mexico \\ ${ }^{4}$ Department of Plastic Surgery, Dr. Ruben Leñero Hospital, Mexico City, Mexico
}

\begin{abstract}
We report the clinical case of a 76- year-old patient who was admitted to the emergency department due to respiratory distress. He had a history of Systemic arterial hypertension, type 2 diabetes mellitus and hypothyroidism. The patient reports symptoms that began three days prior to admission, including dyspnea, fever, myalgia, arthralgia, headache and respiratory distress that was exacerbated 1 day prior to admission. Physical examination revealed a conscious patient with the following vital signs: blood pressure $120 / 62 \mathrm{mmHg}$, heart rate $109 \mathrm{bpm}$, respiratory rate $28 \mathrm{rpm}$, temperature $38.8^{\circ} \mathrm{C}$, arterial oxygen saturation $76 \%$, dyspnea, use of accessory muscles, tachycardia, tachypnea, which met the operational definition for Covid-19. Upon admission to the service, a pulmonary ultrasound was performed in which subpleural consolidations were identified, predominantly in the right hemithorax, as well as an air bronchogram and little pleural effusion. Based on these observations, the patient was admitted to the unit, where management with supplemental oxygen was started using a face mask with a reservoir bag. A chest X-ray was performed in which a diffuse interstitial alveolar pattern was identified in both hemithoraxes. Later a simple chest tomography was performed in which changes compatible with CO-RADS 5 were identified. He continued with established management and during his stay in the service, he evolved to deterioration to need advanced management via air. This study suggests that pulmonary ultrasound may be an effective and reliable tool in the initial evaluation of patients with respiratory distress in the context of Covid-19 pneumonia and that the identification of subpleural consolidations represent a prognostic factor of the disease: identifying them quickly after admission suggests a worse evolution of the disease, thus prompting us to make correct and concrete decisions regarding the management and treatment of these patients, without having to wait for complementary studies such as chest radiography and even tomography.
\end{abstract}

Keywords: Pneumonia, Covid 19, Pulmonary Ultrasound, Chest Tomography, subpleural consolidation

\section{Introduction}

Lung ultrasound has multiple advantages over computed tomography and x-ray equipment. In particular, the $9-15 \mathrm{MHz}$ high-frequency linear array probe can clearly show the morphology and changes of subpleural lesions, and changes in air and water content in consolidated peri pulmonary tissues. ${ }^{1}$ Pulmonary ultrasound in light of current evidence is mainly aimed at the diagnostic orientation of the most frequent causes of acute respiratory failure ( $90.5 \%$ of admission to the emergency room/Intensive Care Unit): pneumothorax, pneumonia, pulmonary consolidations, pulmonary embolism, pulmonary congestion and interstitial disorders, even with superior performance to chest radiography and in this time of pandemic it is an indispensable, useful, easy-to-use tool in the diagnosis, treatment and prognosis of the patient. ${ }^{2,3}$
Quick Response Code:

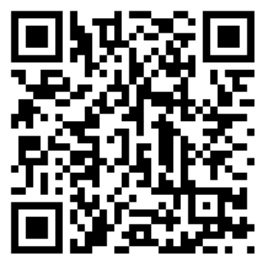

\author{
*Corresponding author: Karen Itzel González Martínez, General Hospital of Ecatepec Las \\ Américas, Simon Bolivar Street/ N, Fraccionamiento Las Americas, Ecatepec, State of México, \\ Mexico \\ Received: 08 April, 2021 \\ Published: 03 May, 2021 \\ Citation: Karen IGM, Felipe JM, Roberto EIÁ, Giovanni CS. Ultrasonically Detected Subpleural \\ Lung Consolidations in The Emergency Room: A Case Report. SOJ Complement Emerg Med. \\ 2021;1(1):1-4. DOI: 10.53902/SOJCEM.2021.01.000505
}




\section{Clinical Case}

Seventy-six years-old patient entering the emergency department for respiratory distress. He had a history of importance for the current condition Systemic arterial hypertension, type 2 diabetes mellitus and hypothyroidism. The current illness began three days prior to admission with dyspnea, fever, myalgia, arthralgia and headache, respiratory distress that was exacerbated 1 day prior to admission. Physical examination revealed a conscious patient with vital signs: blood pressure $120 / 62 \mathrm{mmHg}$, heart rate $109 \mathrm{bpm}$, respiratory rate $28 \mathrm{rpm}$, temperature $38.8^{\circ} \mathrm{C}$, arterial oxygen saturation $76 \%$, dyspnea, use of muscles accessories, tachycardia, tachypnea, met the operational definition for Covid-19. As part of the evaluation in the ABCD emergency department, the patient was transferred to shock air, continuous monitoring was placed and supplemental oxygen with a mask with a reservoir bag at 10 liters/ minute, laboratory studies were requested, pulmonary ultrasound, radiography of chest and simple chest tomography, treatment was started for suspected viral pneumonia secondary to infection by SARS-VOC 2. Causes of acute respiratory failure were ruled out.

Laboratory studies: $\mathrm{Hb} 11.2 \mathrm{~g} / \mathrm{dl}$, Hto 33\%, platelets 220,000, leukocytes 3400, Glucose $366 \mathrm{mg} / \mathrm{dl}$, urea $53 \mathrm{mg} / \mathrm{dl}$, creatinine $1.9 \mathrm{mg} / \mathrm{dl}$, triglycerides $325 \mathrm{mg} / \mathrm{dl}$, total bilirubin $0.5 \mathrm{mg} / \mathrm{dl}$, AST 20U/L, ALT 13UL, GGT 65U/L, FA 65U/L, DHL 268 U/L, Na 140 $\mathrm{mmol} / \mathrm{l}, \mathrm{K} 5.5 \mathrm{mmol} / \mathrm{l}$, chlorine $104 \mathrm{mmol} / \mathrm{l}, \mathrm{Mg} 2.4 \mathrm{mg} / \mathrm{dl}$, TP 12.2sec, TTP 36.1sec, INR 1.1\%, Dimero D 6812ng/ml.

Arterial blood gases: $\mathrm{pH} 7.24, \mathrm{PCO} 264.1 \mathrm{mmHg}, \mathrm{PO} 2$ 63.7mmHg, HCO3 27.4mmol/l, SO2 92\% lactate $1.90 \mathrm{mmol} / \mathrm{l}$.

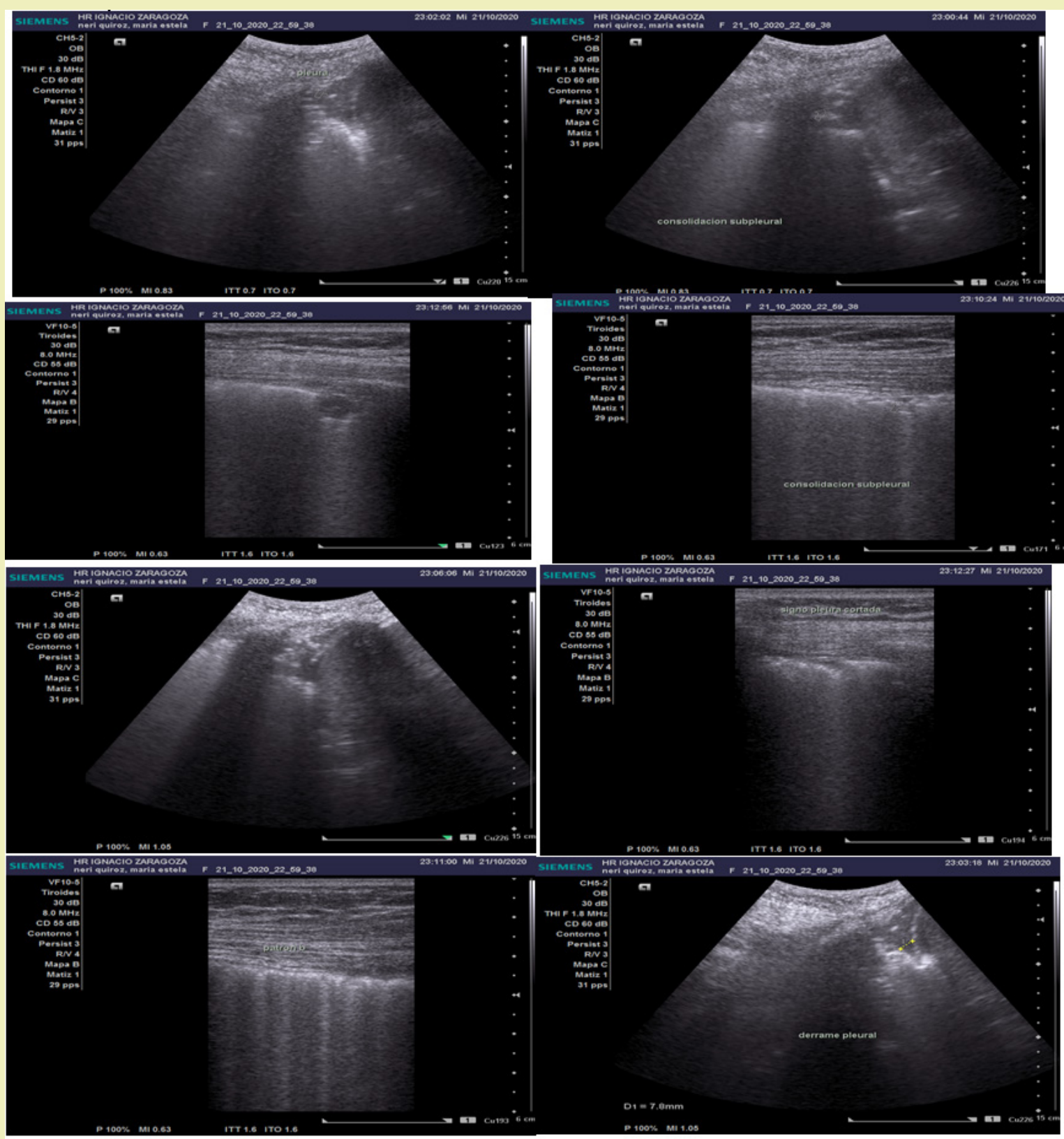

Figure 1: A Subpleural consolidation, B Air bronchogram, C Symptom of the cut pleura, D Lines B, E Scant pleural effusion. 
Pulmonary ultrasound revealed predominantly right subpleural lung consolidations (Figure 1), air bronchogram Figure 1(B), cut pleural sign Figure 1(C), more than 3 B lines Figure 1(D) and little pleural effusion Figure 1(D). Figure 1(E) right, data suggestive of Covid-19. The chest X-ray revealed a diffuse interstitial alveolar pattern in both hemithoraxes (Figure 2). Simple chest tomography reported tomographic images compatible with CO-RADS 5 (very high suspicion of sars-cov 2 infection), with a severity scale due to severe extension, with a score of 22/25 (right lung: $4+4+5$, left lung: $4+5$ ), category a (predominant ground glass pattern), vascular thickening, subpleural consolidations and air bronchogram (Figure 3).

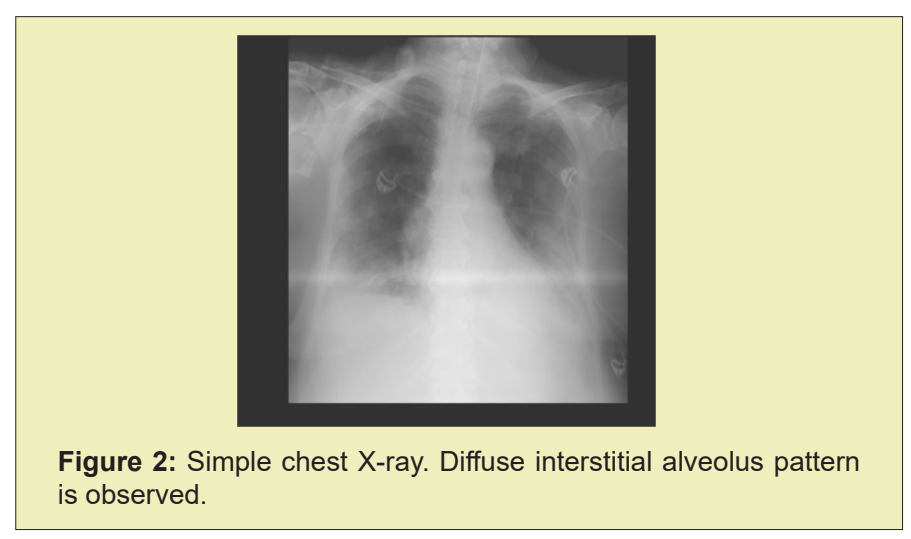

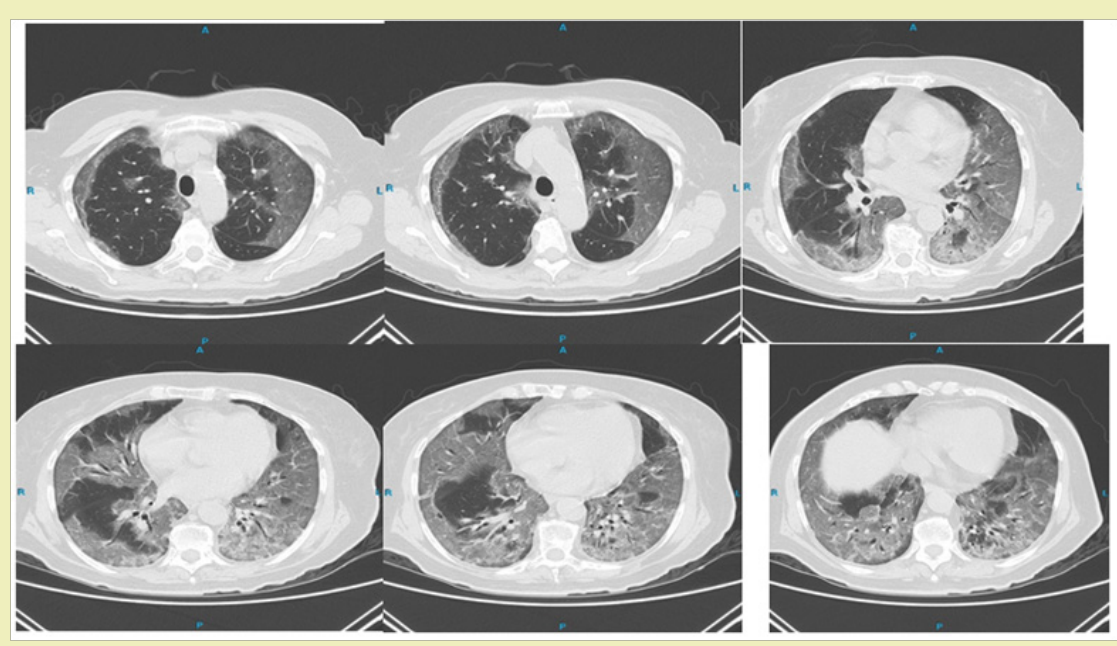

Figure 3: Tomographic mages compatible with CO-RADS 5.

The final diagnosis was Viral Pneumonia secondary to SARSCOV 2 infection, which was confirmed by RT-qPCR on 10-20-2020. Management started with supplemental oxygen, initially with a face mask but due to deterioration and greater respiratory compromise, oxygenation was performed in advanced airways, with non-invasive continuous monitoring, support measures, analgesic, antipyretic and thromboprophylaxis. Heterogeneous parenchyma secondary to areas with a ground glass pattern, which is the predominant pattern, associated with a crazy paving pattern, vascular thickening, subpleural consolidations, and air bronchogram.

\section{Discussion}

Ultrasound findings can usually be visualized early, much earlier than conventional radiological studies. It should be noted that ultrasound is much more sensitive than radiography; and possibly if diagnosed in many patients, especially young mild/moderate cases, which high risk of yielding a false negative chest X-Ray result. Precisely for this reason, ultrasound may be especially useful in primary care where a significant percentage of patients do may not be admitted based on severity criteria, so the findings will have to be assessed accordingly (for example: patient clinically well without alarm criteria, but with consolidations subpleural, which are a serious ultrasound finding that probably requires hospitalization). ${ }^{4,5}$

Pulmonary ultrasound has become a valuable tool for the management of patients affected by COVID-19, not only at the hospital level, but also in Primary Care, as well as for making therapeutic decisions and evaluating the evolution of the disease. Auscultation does not contribute much to the risk posed to the professional, while radiology does not detect subpleural condensations that are small and access to CT in the current situation of sanitary collapse has become highly limited. In addition to being a quick, innocuous and low-cost procedure that can be performed at the patient's bedside, ultrasound is a cost and time-effective technique that is safe for both the patient and the practitioner and allows for better management and even prognosis of the disease.

A common finding in COVID-19 patients is the visualization of subpleural consolidations, which can be identified through the focal interruption of the pleural line. When the consolidation is of greater extent, the lung parenchyma can acquire a sonographic appearance similar to healthy liver parenchyma and may exhibit static or mobile hyperecogenic images corresponding to the dynamic aerial bronchogram described in $\mathrm{CT}^{2}$ Subpleural consolidations initially appear in basal areas and later, may be generalized to the entire lung and gradually increase in size if it keeps progressing the disease There are little things as hypoechoic areas in the pleura base and mobilize thebreathing. They have an irregular deep boundary, are surrounded by B lines and may have an aerial or liquid bronchogram. They are a high-risk ultrasound of complication. If they appear in previous areas they are a sign of poor prognosis..$^{6-8}$ 


\section{Acknowledgments}

None.

\section{Funding}

None.

\section{Declaration of Interest}

Author declares that there is no conflict of interest.

\section{References}

1. Lichtenstein D. Novel approaches to ultrasonography of the lung and pleural space: where are we now? Breathe. 2017;13:100-111.

2. Lichtenstein DA, Peyrouset O. Is lung ultrasound superior to CT? The example of a CT occult necrotizing pneumonia. Intensive Care Med. 2006;32:333-335.
3. Fernandez Bussy S, Labarca G, Lanza M. Thoracic applications of ultrasound. Rev Med Chile. 2016;144:903-909.

4. González Castro A, Escudero Acha P, Peñasco Y, et al. Intensive care during the 2019 coronavirus epidemic Med. Intesiv. 2020;44(6):351362.

5. Zalaquett Eugenio, Lomoro Pascal, Natalizi Anna. Pulmonary Ultrasound in COVID-19. Rev Chil Radiol. 2020;26(2):46-51.

6. Peng QY, Wang XT, Zhang LN. Chinese Critical Care Ultrasound Study Group (CCUSG). Finding of lung ultrasonography of novel coronavirus pneumonia during the 2019-2020 epidemic. Intensive Care Med. 2020.

7. Villén T, Cheyayeb J, Campo R. Pulmonary Ultrasound for the Management of COVID-19 Infection. Direct from SEMES. 2020.

8. Rincón Salas JJ. Manual of ultrasound in intensive care and emergencies. $2^{\text {nd }}$ edn. Mexico, 2016:41-57. 